\title{
A PERFECT RECONSTRUCTION FILTER BANK WITH IRRATIONAL DOWN-SAMPLING FACTORS
}

\author{
Soo-Chang Pei, Meng-Ping Kao, and J. J. Ding \\ Department of Electrical Engineering \\ National Taiwan University \\ Taipei, Taiwan 10617, ROC \\ E-mail: pei@cc.ee.ntu.edu.tw, Fax: 886-2-23671909
}

\begin{abstract}
Most of the existing methods designed to implement fractional interpolation and decimation are limited by rational scaling factors such as $L / M$, where $L$ and $M$ are positive integers. The general procedure is usually done with up-sampling by $L$ first, and then followed by down-sampling by $M$. This scheme, however, requires two steps to fulfill and is not capable in dealing with irrational scaling factors, which can not be represented by $L / M$. In this paper, we propose a new method to solve the above two difficulties by a single step. Furthermore, a perfect reconstruction filter bank is derived using this new method and the experimental results are presented.
\end{abstract}

Index terms-irrational scaling, interpolation, decimation, nonuniform filter bank, perfect reconstruction

\section{I . INTRODUCTION}

In a conventional filter bank system, the scaling operation is very important in that it controls the sampling rate to meet the system requirements. However, as long as a discrete time system is considered, most previous works fail to realize a scaling operation with irrational factors. For example, given a discrete time signal $x[n]$, how can we scale it by $\sqrt{2}$ ? Although it seems quite difficult to be realized in time domain, Rao [1]-[3] developed a practical method to accomplish it using the concept of frequency domain warping. This method, however, requires the computation of inverse discrete-time Fourier transform (IDTFT) which lacks a closed form formula to avoid the computational approximation. Furthermore, it is not well suitable in a perfect reconstruction (PR) filter bank in practice.

Partly motivated by the frequency warping concept, we propose a new method devoted to maintaining the linearity while doing the frequency warping. Fortunately, this leads to a concise closed form formula in time domain, which is invulnerable to any computational approximation.
It also works very well in the proposed PR filter bank structure.

The paper is organized as follows. Section II provides the derivation of the new scaling method and the structure of the irrational scaling PR filter bank is shown in Section III. Section IV presents the simulation results of the above scheme. Finally the conclusion is made in Section V.

\section{II . PROPOSED IRRATIONAL SCALING ALGORITHM}

Given a discrete time signal $x[n]$, suppose its scaled version is $x_{a}[n]$, where $a$ is the scaling factor and $a \in \mathfrak{R}$, $a>0$. In this way, $a>1$ is equivalent to interpolation and $0<a<1$ is for decimation. Referring to the scaling property of continuous time Fourier transform (CTFT) as below,

$$
\begin{gathered}
x(t) \stackrel{\text { CTFT }}{\longleftrightarrow} X(j \omega) \\
x\left(\frac{t}{a}\right) \stackrel{\text { CTFT }}{\longleftrightarrow}|a| X(j \omega a)
\end{gathered}
$$

we could reasonably infer (1) to its DTFT counterpart, i.e.

$$
\begin{gathered}
x[n] \stackrel{\text { DTFT }}{\longleftrightarrow} X\left(e^{j \omega}\right) \\
x_{a}[n] \stackrel{\text { DTFT }}{\longleftrightarrow} a \mid X\left(e^{j \omega a}\right)
\end{gathered}
$$

Because $X\left(e^{j \omega}\right)$ is a continuous time function with period $2 \pi, X\left(e^{j \omega a}\right)$ by definition exists whenever $a \in \mathfrak{R}$.

$$
X\left(e^{j \omega}\right)=\sum_{n=-\infty}^{\infty} x[n] e^{-j \omega n}
$$

By (2), 


$$
\begin{aligned}
& x_{a}[n]=\operatorname{IDTFT}\left\{a X\left(e^{j \omega a}\right)\right\} \\
& =\frac{1}{2 \pi} \int_{-\pi / a}^{\pi / a} a X\left(e^{j \omega a}\right) e^{j \omega n} d \omega \\
& =\frac{1}{2 \pi} \int_{-\pi / a}^{\pi / a} a \sum_{k=-\infty}^{\infty} x[k] e^{-j \omega \omega k} e^{j w n} d w \\
& =\frac{a}{2 \pi} \sum_{k=-\infty}^{\infty} x[k] \int_{-\pi / a}^{\pi / a} e^{j(n-a k) \omega} d \omega
\end{aligned}
$$

We now divide (3) into 2 disjoint cases, ones being $k=n / a$ and the others being $k \neq n / a$. For $k=n / a$,

$$
\int_{-\pi / a}^{\pi / a} e^{j(n-a k) \omega} d \omega=\int_{-\pi / a}^{\pi / a} d \omega=\frac{2 \pi}{a}
$$

For $k \neq n / a$,

$$
\int_{-\pi / a}^{\pi / a} e^{j(n-a k) \omega} d \omega=\frac{2 \pi}{a} \operatorname{sinc}\left(\frac{n}{a}-k\right)
$$

From (3),(4) and (5),

$$
\begin{aligned}
x_{a}[n]= & \frac{a}{2 \pi}\left[\sum_{k=-\infty, k=n / a}^{\infty} x[k] \int_{-\pi / a}^{\pi / a} e^{j(n-a k) \omega} d \omega\right. \\
& \left.+\sum_{k=-\infty, k \neq n / a}^{\infty} x[k] \int_{-\pi / a}^{\pi / a} e^{j(n-a k) \omega} d \omega\right] \\
= & \sum_{k=-\infty, k=n / a}^{\infty} x[k]+\sum_{k=-\infty, k \neq n / a}^{\infty} x[k] \operatorname{sinc}\left(\frac{n}{a}-k\right) \\
= & \sum_{k=-\infty}^{\infty} x[k] \operatorname{sinc}\left(\frac{n}{a}-k\right)
\end{aligned}
$$

Note that we have changed the upper bound and lower bound of the definite integral of (3) from $[-\pi,+\pi]$ to $[-\pi / a,+\pi / a]$ for the purpose of removing the high frequency spectrum. The unwanted spectrum is introduced when scaling a periodic signal to a different new period, which is, in our case, from $2 \pi$ to $2 \pi / a$. In other words, changing the integral upper/lower bound is equivalent to applying an ideal low-pass filter after up-sampling. We also ignore the absolute value of $|a|$ because $a>0$ throughout this paper. Eq.(6) is only suitable in the case where $a>1$, i.e. interpolation.

For the case where $0<a<1$, i.e. decimation, the upper and lower bound should be changed back to $[-\pi,+\pi]$ again to avoid the aliasing due to down sampling. This is equivalent to applying an ideal low-pass filter before down-sampling.

$$
\begin{aligned}
& x_{a}[n]=\operatorname{IDTFT}\left\{a \quad X\left(e^{j \omega a}\right)\right\} \\
& =\frac{a}{2 \pi} \sum_{k=-\infty}^{\infty} x[k] \int_{-\pi}^{\pi} e^{j(n-a k) \omega} d \omega=a \sum_{k=-\infty}^{\infty} x[k] \operatorname{sinc}(n-a k)
\end{aligned}
$$

For convenience, we summarize the above two formulas in Table 1.
Table 1 Irrational scaling formulas

\begin{tabular}{|c|c|c|}
\hline Interpolation & $\mathrm{a}>1$ & $\sum_{k=-\infty}^{\infty} x[k] \operatorname{sinc}\left(\frac{n}{a}-k\right)$ \\
\hline Decimation & $\mathrm{a}<1$ & $a \sum_{k=-\infty}^{\infty} x[k] \operatorname{sinc}(n-a k)$ \\
\hline
\end{tabular}

This is not the only way to derive irrational scaling formulas. Adams [4] has given a different approach to do irrational interpolations from the view point of sincinterpolated discrete time signals. However, this method has difficulties in doing irrational decimations without aliasing. It is a surprise that after a modification of the down-sampling counterpart, identical formulas can be achieved as those shown in Table 1. The details are described in Appendix.

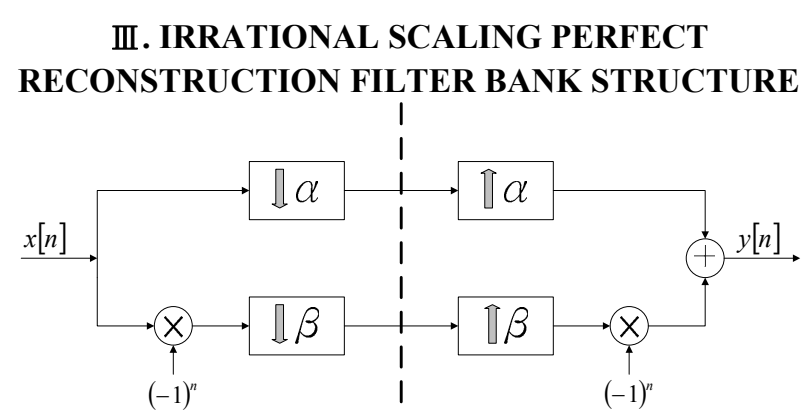

Fig.1 Irrational scaling perfect reconstruction filter bank structure

The fundamental structure of the PR filter bank using irrational scaling factors is shown in Fig.1, where $\alpha, \beta \in R, \alpha, \beta>1$, and $1 / \alpha+1 / \beta=1$. The basic goal is to perfectly reconstruct the output such that $y[n]=x[n]$ under any circumstances, even if $\alpha$ and $\beta$ are irrational numbers.

In Fig.1, the up sampling and down sampling kernel is almost identical to the results in Section $\Pi$, except for small modifications by replacing $a$ by $1 / a$ in the decimation case, in which we rearrange in Table 2.

Table 2 Irrational up and down sampling kernels

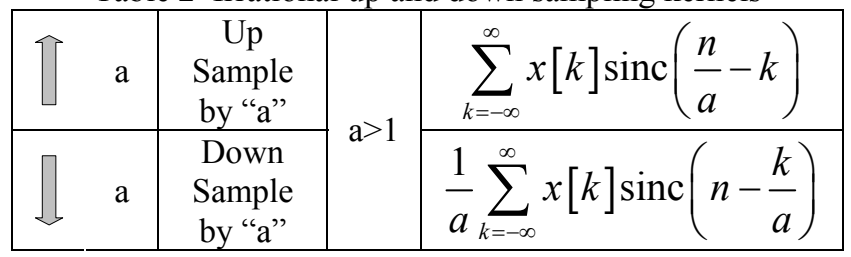




\section{EXPERIMENTAL RESULTS}

To test the proposed PR FB structure in Fig.1, we take a 256 points normalized Gaussian random signal with zero mean and unit variance as input sequence $x[n]$, choosing $\alpha_{1}=\sqrt{2}$ and $\alpha_{2}=2+\sqrt{2}$ to construct a twochannel non-uniform PR FB. Since the index $k$ in Table II has a range from minus infinity to infinity which is not feasible in practical implementations, here we choose $k=[-256,+256]$ in our experiment and observe the reconstruction errors due to truncation of $k$. After reconstruction, the mean absolute value of the reconstruction error is only 0.0026 , which is shown in Fig.2(e). Note that these errors are introduced by truncation of $k$, which makes the equivalent low-pass filters no ideal any more. However, the errors keep decreasing as $k$ grows larger and the filter bank structure virtually becomes perfect reconstruction as $k \rightarrow \infty$

Although the above formulas are designed for onedimensional signals, they could be well extended to work with two-dimensional images via two separable scalings in horizontal and vertical directions. The modified structure is shown in Fig.3 for the 4-channel non-uniform PR FB case.

As a result, we take a gray level $256 * 256$ "Lena" image as an input, choosing $\alpha_{x}=\sqrt{3}, \beta_{x}=\frac{3+\sqrt{3}}{2}$ and $\alpha_{y}=\sqrt{2}$, $\beta_{y}=2+\sqrt{2}$ to produce a rectangular down sampled image, and using the system in Fig. 3 to reconstruct the original image perfectly. The results are shown in Fig.4. As we can see, the down sampled image in the upper channel is of $182 * 148\left(\left\lceil\frac{256}{\sqrt{2}}\right\rceil *\left\lceil\frac{256}{\sqrt{3}}\right\rceil\right)$ resolutions. The reconstructed image is of $256 * 256$ resolutions and is almost identical to the original image. The mean absolute value of the reconstruction error is about 0.05 .

\section{CONCLUSION}

In this paper, a perfect reconstruction filter bank using irrational scaling factor is proposed. The advantage of the new scaling algorithm is that it is a concise closed form formula in time domain, and it completes an irrational scaling in a single step with removing unwanted images and avoiding aliasing simultaneously. The experimental results have shown the perfect reconstruction after a several irrational scalings.

\section{APPENDIX}

At first, we consider $x[n]$ as a non-aliasing sampled version of a continuous time signal $x(t)$, i.e.

$$
\begin{aligned}
x(t) & =\sum_{n=-\infty}^{\infty} x[n] \frac{\sin [\pi(t-n T) / T]}{\pi(t-n T) / T} \\
& =\sum_{n=-\infty}^{\infty} x[n] \operatorname{sinc}(t-n)
\end{aligned}
$$

where we have assumed the sampling period $T=1$. Choosing the new sampling period $T^{\prime}=1 / a$, we get

$$
\begin{aligned}
x_{a}[n] & =x\left(\frac{n}{a}\right) \\
& =\sum_{k=-\infty}^{\infty} x[k] \operatorname{sinc}\left(\frac{n}{a}-k\right)
\end{aligned}
$$

For $a>1,(8)$ is the same as $(6)$. On the other hand, as for $a<1$, we pre-filter $x(t)$ with an ideal low pass filter $H(j \omega)$ to avoid the aliasing effect caused by decimation, where

$$
\begin{aligned}
& H(j \omega)=\left\{\begin{array}{c}
1, \quad \text { when }|\omega| \leq a \pi \\
0, \text { else }
\end{array}\right. \\
& h(t)=\operatorname{ICTFT}\{H(j \omega)\}=a \operatorname{sinc}(a t)
\end{aligned}
$$

is the impulse response of $H(j \omega)$.

$$
\begin{aligned}
& x_{L P}(t)=x(t) \otimes h(t) \\
& =\left\{\sum_{n=-\infty}^{\infty} x[n] \operatorname{sinc}(t-n)\right\} \otimes h(t) \\
& =\sum_{n=-\infty}^{\infty} x[n]\{\operatorname{sinc}(t-n) \otimes h(t)\} \\
& =a \sum_{n=-\infty}^{\infty} x[n] \operatorname{sinc}(a t-a n)
\end{aligned}
$$

where $x_{L P}(t)$ denotes the low pass filtered signal of $x(t)$. The last step is to re-sample $x_{L P}(t)$ with new period $T^{\prime}=1 / a$, i.e.

$$
\begin{aligned}
& x_{a}[n]=x_{L P}\left(\frac{n}{a}\right) \\
= & a \sum_{k=-\infty}^{\infty} x[k] \operatorname{sinc}(n-a k)
\end{aligned}
$$

Note that (9) is the same as (7), which completes the derivation of the alternative approach.

\section{REFERENCES}

[1] W. Zhao and R.M. Rao, "Discrete-time continuous-dilation wavelet transforms," Proceedings of the IEEE-SP International Symposium on Time-Frequency and Time-Scale Analysis, Oct 1998, Page(s): 233 $-236$

[2] W. Zhao, R.M. Rao, "Continuous-dilation discrete-time self-similar signals and linear scale-invariant systems," Proceedings of the 1998 
IEEE International Conference on Acoustics, Speech, and Signal Processing, Volume: 3, Page(s): 1549 -1552

[3] Seungsin Lee, W. Zhao, R. Narasimha and R.M. Rao, "Discrete-time models for statistically self-similar signals," IEEE Transactions on Signal Processing,
Volume: 51, Issue: 5, May 2003, Page(s): 1221 -1230

[4] J.W. Adams, R.W. Bayma, and J.E. Nelson, ”Digital Filter Design for Generalized Interpolation," IEEE International Symposium on Circuits and Systems, May 1989, Page(s): 1299 -1302 vol.2

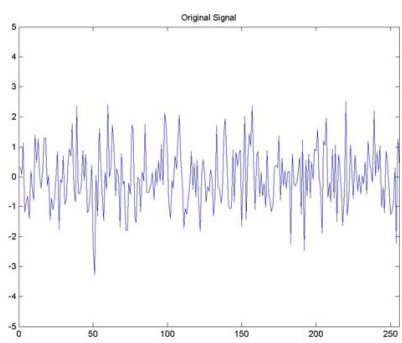

(a)

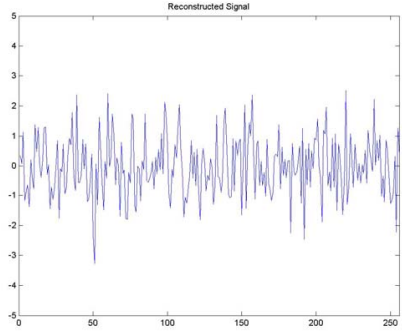

(d)

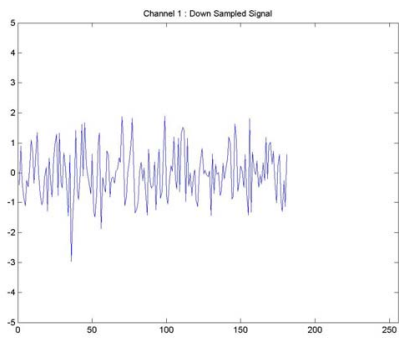

(b)

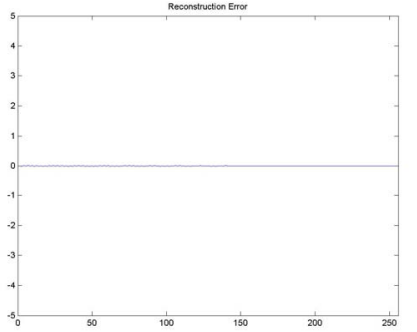

(e)

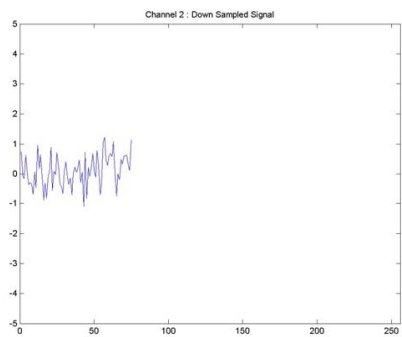

(c)

Fig. 2 (a)Original input signal.

(b)Down sampled $\alpha=\sqrt{2}$ signals. (c)Down sampled $\beta=2+\sqrt{2}$ signals. (d)Perfectly reconstructed output signal. (e)Reconstruction error.

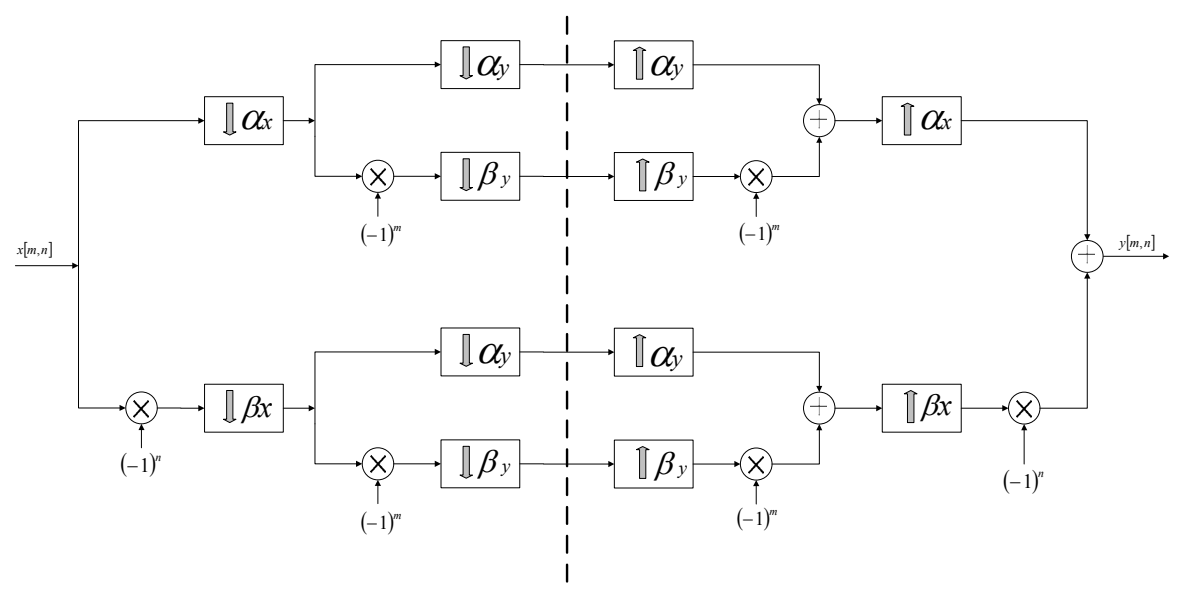

Fig.3 2D PR filter bank structure

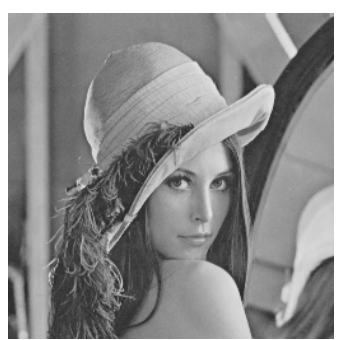

(a)

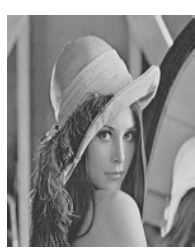

(b)

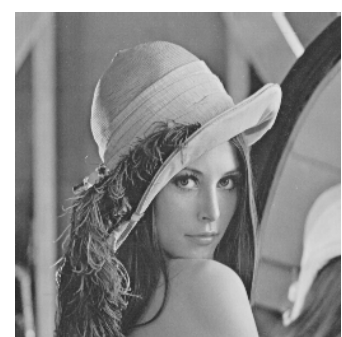

(c)

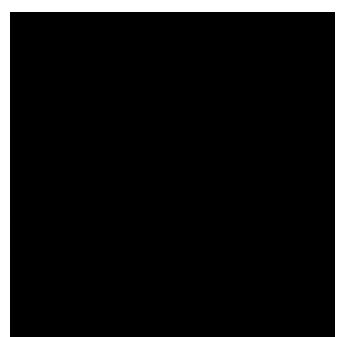

(d)

Fig.4 (a) Original input image. (b) Down sampled image in the upper channel.

(c) Perfectly reconstructed output image. (d) Reconstruction error. 